\title{
Temporal evolution of chromospheric downflows
}

\author{
Andreas Lagg, J. Woch, N. Krupp, A. Gandorfer, S. K. Solanki \\ Max Planck Institute for Solar System Research, 37191 Katlenburg-Lindau, Germany. \\ email: lagg@mps.mpg.de
}

\begin{abstract}
At the footpoints of loops spanning a site of flux emergence, earlier investigated in the papers by Solanki et al. (2003) and Lagg et al. (2004), we find large redshifts in the He $1083 \mathrm{~nm}$ line coexisting with an almost unshifted component. The speed associated with these redshifts reaches values as high as $40 \mathrm{~km} / \mathrm{s}$. We interpret these downflows in the context of several models: the free-fall downflow of matter along vertical field lines (Schmidt et al. 2000), the redshift by downward propagating acoustic waves (Hansteen 1993) and the motion of condensation regions to either side of loop footpoints (Müller et al. 2003). We present the temporal evolution of these redshifts and reconstruct the magnetic field vector in these regions for both the redshifted and the unshifted atmospheric component.
\end{abstract}

\section{Observation and Analysis Technique}

An active region in the course of emergence (NOAA 9451), located at $33^{\circ} \mathrm{W}, 22^{\circ}$ $\mathrm{S}(\mu=\cos \Theta=0.8)$, was recorded with the Tenerife Infrared Polarimeter (TIP) on the Vacuum Tower Telescope at the Teide observatory on Tenerife. The spectral resolution was $30 \mathrm{~m} \AA$ per pixel, the spatial resolution was limited by the seeing $\left(\approx 1.5^{\prime \prime}\right)$. The temporal evolution of the active region was covered by repetitive scans over an interval of 73 minutes. The exposure time of $\approx 5$ s per slit position led to a noise level of typically $\approx 5 \cdot 10^{-4}$. The observed wavelength range from 1082.5 to $1083.3 \mathrm{~nm}$ contains two photospheric lines of Si I and Ca I, the chromospheric He I multiplet (eff. Landé factors 2.0, 1.75 and 1.25) and a telluric blend at $1083.2 \mathrm{~nm}$ (see eg. Rüedi et al. 1995).

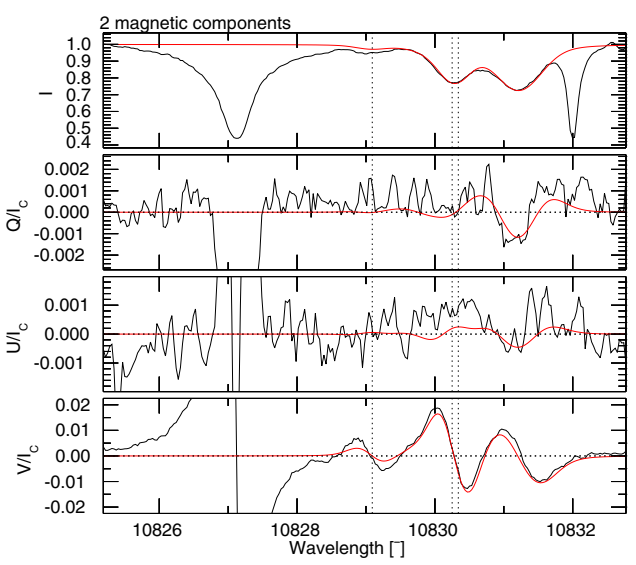

Figure 1. Profile showing 2 magnetic components (observed profile: black, fitted profile: red). Fit parameters for fast component: $\gamma=$ $65^{\circ}, \mathrm{B}=640 \mathrm{G}$; component at rest: $\gamma=26^{\circ}$, $\mathrm{B}=470 \mathrm{G}$

The He I triplet, which has a complex non-LTE line formation but is nearly optically thin, was analyzed by applying an inversion based on the Unno-Rachkowski solution to describe the individual Zeeman components of each member of the triplet to the data (Rachkowski 1967) combined with a simple, empirical implementation of the Hanle effect (method described in Lagg et al. 2004; Solanki et al. 2003). The inversion allows us to retrieve the full magnetic vector in the upper chromosphere, where the He I triplet is formed. Additionally we obtain line-of-sight-velocity maps for the observed regions. 


\section{Results and Interpretation}

The obtained maps for the magnetic field vector strongly indicate the presence of closed magnetic loops over the site of flux emergence (Solanki et al. 2003). At both footpoints of these loops the Stokes profiles suggest the presence of a fast downflow component (LOS-velocity up to $40 \mathrm{~km} / \mathrm{s}$ ) and an unshifted component within one image element (see Fig. 1). This downflow is present over the whole interval covered by the observations (i.e. 73 minutes). Close to, but not exactly at the position of the fastest downflow we often observe the He I line in emission.

For the pixels where the fast downflow component is clearly separated from the unshifted component we were able to determine the magnetic field vector for both components independently (see Fig. 1). The fast component is less inclined $\left(50^{\circ}<\gamma<80^{\circ}\right)$ than the slow component $\left(\gamma<30^{\circ}\right)$. The magnetic field strength for both components is comparable.

We interpret the fast downflow as a free fall of matter along loop field lines (Schmidt et al. 2000). The downflow is caused by the draining of loops as they rise into higher, less dense layers. Other interpretations of large redshifts, like acoustic waves (Hansteen 1993) or moving condensation regions (Müller et al. 2003) are not consistent with the observed signature. The first model fails to reproduce the high velocities, the second model results in a periodic downflow to one of the loop footpoints. However, the upward motion of the loop apex in our observations is not fulfilling the assumption of Müller et al. (2003) of a steady-state loop.

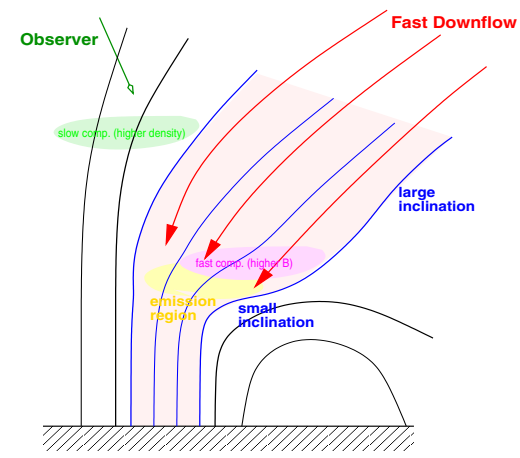

Figure 2. Possible magnetic field topology of observed region.

The magnetic field configuration for the two unresolved components can be explained by two different models: either the chromospheric field is "uncombed", i.e. fibril-like structures with different magnetic field inclinations (flux tubes with sizes $<400 \mathrm{~km}$ ) coexist within a resolution element, or the absorption signal in He I is produced by two different layers in the solar atmosphere (see sketch in Fig. 2).

\section{References}

Hansteen, V. (1993), A new interpretation of the redshift observed in optically thin transition region lines, ApJ, 402, 741-755.

Lagg, A., J. Woch, N. Krupp, and S. K. Solanki (2004), Retrieval of the full magnetic vector with the He I multiplet at $1083 \mathrm{~nm}$. Maps of an emerging flux region, A\&A, 414, 1109-1120.

Müller, D. A. N., V. H. Hansteen, and H. Peter (2003), Dynamics of solar coronal loops. I. Condensation in cool loops and its effect on transition region lines, $A \mathscr{E} A$, 411, 605-613.

Rachkowsky, D. N. (1967), The reduction for anomalous dispersion in the theory of the absorption line formation in a magnetic field (in Russian), Izv. Krym. Astrofiz. Obs., 37, 56-61.

Rüedi, I., S. K. Solanki, and W. C. Livingston (1995), Infrared lines as probes of solar magnetic features. X. HeI 10830A as a diagnostic of chromospheric magnetic fields., A $\& A$, 293, 252262.

Schmidt, W., K. Muglach, and M. Knölker (2000), Free-fall Downflow Observed in He I 1083.0 Nanometers and $\mathrm{H} \beta, A p J, 544,567-571$.

Solanki, S. K., A. Lagg, J. Woch, N. Krupp, and M. Collados (2003), Three-dimensional magnetic field topology in a region of solar coronal heating, Nature, 425, 692-695, doi:10.1038/nature02035. 